\title{
DE89 015356
}

INVITED PAPER: XVI INTERNATIONAL CONFERENCE ON THE PHYSICS OF ELECTRONIC AND ATOMJC COLLISIONS, JULY 26 - AUGUST 1, 1989, NEW YORK, NEW YORK

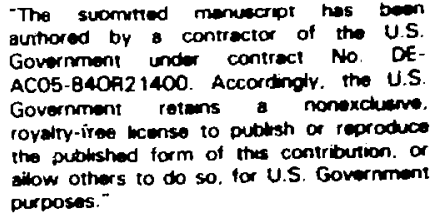

ON THE UTILITY AND UBIQUITY OF ATOMIC COLLISION PHYSICS

Sheldon Datz

Physics Division, Oak Ridge National Laboratory, Oak Ridge, Tennessee 37831-6377, USA

This paper is divided into three parts. In the 1ntroduction, we discuss the history and makeup of ICPEAC. In the second part, we discuss the extent of applicability of atomic collision physics. In the third part, we chose one subject (dielectronic excltation) to show the interrelationship of various sub-branches of atomic collision physics

\section{INTRODUCTION}

Fron the Presace to Book of Abstracts for ICPEAC II (Boulder, Colorado, 1961):

"This conference is the second in a series of informat meetings organized by a group of. workers in the general field of electronis and atomic collisions. The first such meeting was held at New York University in 1958, and we will probably continue to meet at irregubar intervals in the future ...."

\section{Benjamin Bederson Conference Secretary}

This is the thirtieth anniversary, actually the thirty first, of ICPEAC. It all began in New York in 1958 with 70-80 people getting together at New York University for two days to discuss their mutual concerns. There were 47 papers, a book of abstracts, a cocktall party at the Fifth Avenue Hotel, and a good time was had by all. Since that time, the conference has grown by more than a factor of ten.

To refresh your menories, the conferences accurred as follows:

\begin{tabular}{|c|c|c|}
\hline I & New York, NY & 1958 \\
\hline II & Boulder, $C O$ & 1961 \\
\hline III & London, UK & 1963 \\
\hline TV & Quebec, Canada & 1965 \\
\hline$\because$ & Len1ngrad, USSR & 1967 \\
\hline$V:$ & Cambridge, MA & 1969 \\
\hline 35 & Amsterdam, the Netherlancs & $: 971$ \\
\hline IIII & Beograd, Yugoslavia & 1973 \\
\hline IX & Seattle, WA & 197 \\
\hline$y$ & Paris, France & 1977 \\
\hline$\not \mathrm{I}$ & Kyoto, Japan & 1979 \\
\hline SI: & Gatlinburg, TA & !98: \\
\hline ATII & w. Ber11n, FRC & $! 983$ \\
\hline $\begin{array}{l}\text { YIV } \\
\text { XV }\end{array}$ & $\begin{array}{l}\text { Stanford, CA } \\
\text { Brighton, England }\end{array}$ & $\begin{array}{l}1985 \\
1987\end{array}$ \\
\hline XVI & New York, NY & 198 \\
\hline
\end{tabular}

\begin{abstract}
Compltees can vote on pollcles and invited papers, but particlpants vote with their interest, thelr alrplane tickets, and their concributed papers. Fig. I shows the growth of contributed papers ab ovo. The growth is almost continuous with jogs developing at ca 1980, because of the accelerated growth of the field in Europe. In its present form, one might expect an asymptote at $\sim 900-1000$ total contributed papers.
\end{abstract}

Total number of contributed papers at ICPEAC

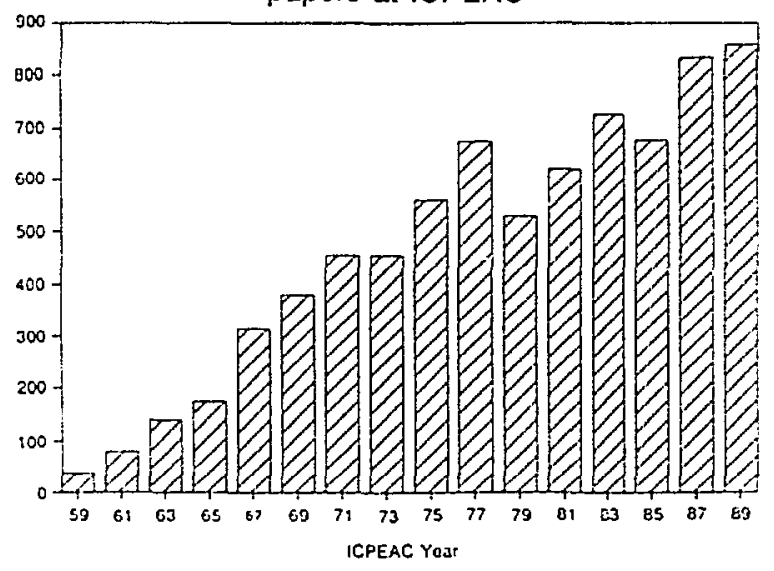

FIG. 1. Total number of rontributed papers at ICPEAC.

In $\overrightarrow{F i g .} 2$, we show the trends in subfields over the last ilx ICPEACs. Taking an even not? decalied look at sub-jiseffilines ard assuning me radical changes in ICPEAC policy uz governmente funding profflas, one might sefely prollet for example:
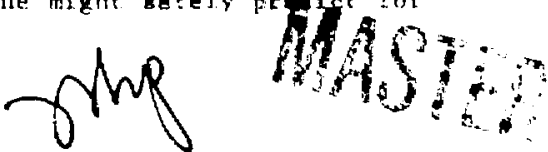
- continued but declining dominance of ion-atom collisions

- steady decline in ion/atom-molecule fraction

- growth in high temperature plasma related flelds, e.g., ion-ton and electron-ion collisions

- growth in collisions of "exot1c" $e^{+}, \vec{p}, \mu^{ \pm}$, etc. spectes

- possible growth in clusters and solid interactions

- continued growth in photon-atom/molecule coll1sions.

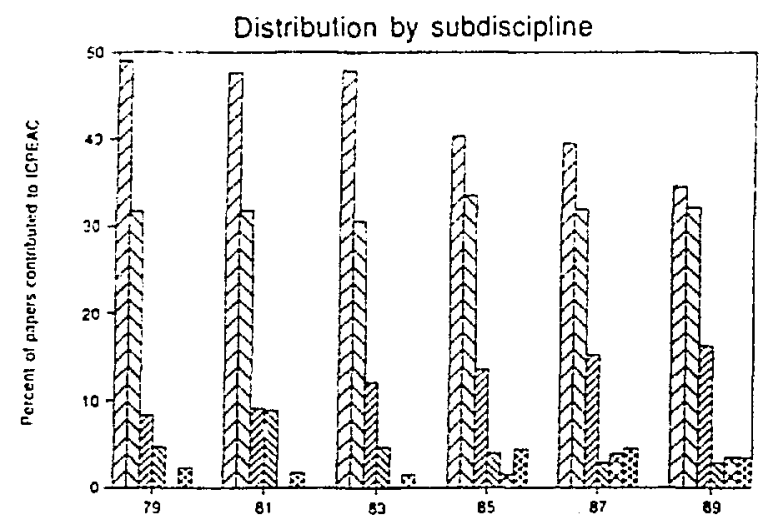

FIG. 2. Distribution of contributed papers among the sub-fields of ICPEAC over the last s1x conferences. 7 ion/atom-atom/mol; DS e-atom/mol.; 272 hv-atom/nol. exotic species;
Rydbergs and field assisted;

This latter point is especially worth noting. Since 1t's humble beginning as two contributed papers in 1965, photon interactions have grown to 130 contributions $(16 \%)$ at the present meeting. This growth, shown in Fig. 2, is correlated with the development of the new tools, lasers and synchrotron light sources. It is further noteworthy that almost a third of this work Involves the interaction of photons and witn molecules; neither of these collision partners is mentioned in the present conference title. Evidently the practitioners in this area feel a strong identification with the community of collision physicists, tather than spectroseodists.

\section{I1. THE APPLICABILITY OF ATOMIC COLLISION PHYSICS}

The need for detalled knowledge of atomic coliision physics permeates many flelde ut science and technology. In nuciear physics, for example, there 1 s a need for information or atopping powers and on the states of energetic lons penetrating solids and gases. 1 In this latter case, the need arises in the study of hyperfine interactions by, e.g., perturbed angular correlations.

\section{A. Condensed Matter Physics}

In condensed matter physics, the applications are very broad. They include methods of surface and near surface characterization and materials modification. A number of these are listed in Table $I$.

You will note that the range of energies used in the atomic probes of solids is quite broad. This breadth is a characteristic of atonic collision physics. In fact, the range exceeds that found in any other branch of sclence. This is 11lustrated in Fig. 3 where we show atomic collision physics related tranches of science and technology as a function of energy; the range covers 16 orders of magnitude in energy!

Actually, if one wishes, one could include even lower energy interactions such as those involving hyperfine state changes in atomic hydrogen which occur at $1420 \mathrm{MHz}=6 \times 10^{-6} \mathrm{eV}(0.1$ cal./mol.) in the interstellar medium. But, instead, I have taken, as a lower base, the mean temperature of the incerstellar medium $10^{\circ} \mathrm{K}$ $10^{-3} \mathrm{eV}$. Since the recent proposal to build THC, a heavy-ion (hadron) collider at CERN, we could extend the upper end to $\sim 10^{15} \mathrm{eV}$ ( $\mathrm{Pb}$ at $4 \mathrm{TeV} / \mathrm{C}$ ) amu).

\section{B. Astrophysics}

The range of atomic collision energies involved in astrophysics covers the entire extent shown in Fig. 3 and, as was pointed out in Alex Dalgarno's ICPEAC XII paper, 3 "Most of the knowledge we have about the universe resides in the form of photons. I'o interpret the message they bring in their jourmey to us, we must reconstruct the events in which they participated... The processes which produce the photons and the processes which modify them belong usually to the domain of electron, atomic, and molecular physics."

In Fig. 4, a picture of the sun taken with radiation at $173 \AA( \pm 1 A)$ is shown. The light emanates mostly from $F e ~ I X$ and $F e X$ in the solar corone at $-10^{E}$ degrees $\left(\sim 10^{2} \mathrm{eV}\right) .^{4}$ पañ features in the corona structure (loops, leveis. atc.) are visible. It is noteworthy here chat discrepancy of a factor of two in the temperature of the golar corona as seasured by the derolty of gradient of the sorou and thr Doppler widths of the spectral ifres and by suundance of Narlike Fe was setrled by Burgess, who introdured dielactronte recom 
Table I. Some Applications of Atomic Collision Physics to Condensed Matter Sclence and Technology. 2

\begin{tabular}{|c|c|}
\hline $\begin{array}{l}\text { Low Energy Ion Scattering and Atomic Diffraction } \\
(0.01 \mathrm{eV}-1 \mathrm{keV})\end{array}$ & Top surface composition and structure. \\
\hline $\begin{array}{l}\text { High Energy Ion Scattering } \\
(100 \mathrm{keV}-10 \mathrm{MeV})\end{array}$ & $\begin{array}{l}\text { Atomic composition of surface and near } \\
\text { surface layers. Lattice location of } \\
\text { impurities in single crystals. }\end{array}$ \\
\hline $\begin{array}{l}\text { Ion-Induced X-Ray Emission } \\
\quad(1-100 \mathrm{MeV})\end{array}$ & $\begin{array}{l}\text { Proton induced } X \text {-ray analysis. heavy ion } \\
\text { induced } X \text {-ray spectra, atomic environment } \\
\text { at lateice site. }\end{array}$ \\
\hline $\begin{array}{l}\text { Secondary Ion Mass Spectrometry } \\
(100 \mathrm{eV}-10 \mathrm{keV})\end{array}$ & $\begin{array}{l}\text { Sputtered ions are analyzed; depth profiling } \\
\text { possible. }\end{array}$ \\
\hline $\begin{array}{l}\text { Photoelectron and Auger Spectroscopy } \\
\qquad(50 \mathrm{eV}-1 \mathrm{keV})\end{array}$ & Surface and near surface compostion. \\
\hline $\begin{array}{l}X \text {-ray Fluorescence Analysis } \\
(100 \mathrm{eV}-100 \mathrm{keV})\end{array}$ & Atomic composition in near surface region. \\
\hline $\begin{array}{l}\text { Macerial Modiffcation; Ion-Implantation } \\
\quad(100 \mathrm{keV}-10 \mathrm{MeV})\end{array}$ & $\begin{array}{l}\text { sodification of mechanical, chemical, and } \\
\text { electronic properties. }\end{array}$ \\
\hline $\begin{array}{l}\text { Microfabrication } \\
\quad(10-100 \mathrm{keV})\end{array}$ & $\begin{array}{l}\text { Photo-, } X \text { ray-, electron beam-, and ton } \\
\text { beam-lithography }\end{array}$ \\
\hline $\begin{array}{l}\text { Radiation Biology } \\
\qquad(1 \mathrm{eV}-10 \mathrm{GeV})\end{array}$ & $\begin{array}{l}\text { Interactions of lonizing radiation and } \\
\text { particles with biological systems (also } \\
\text { heavy ion therapy). }\end{array}$ \\
\hline
\end{tabular}

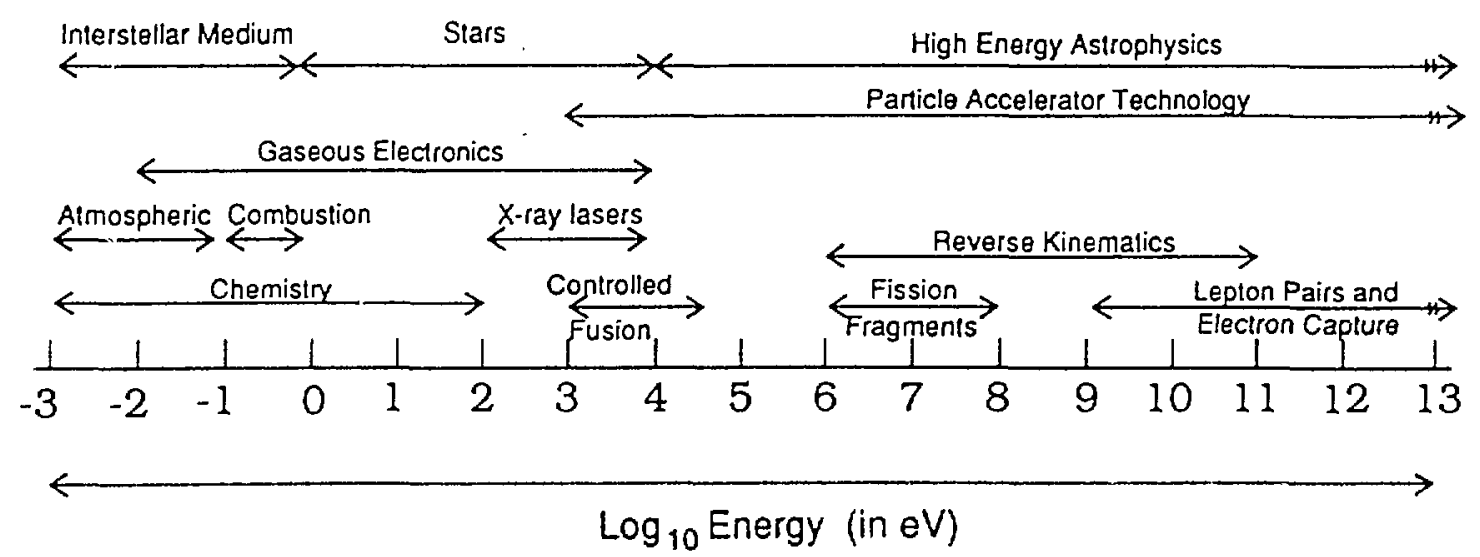

Fig. 3. Some of the sciences and technologles which require acualc colltsion physics input ds a function of the relevant energy range. 


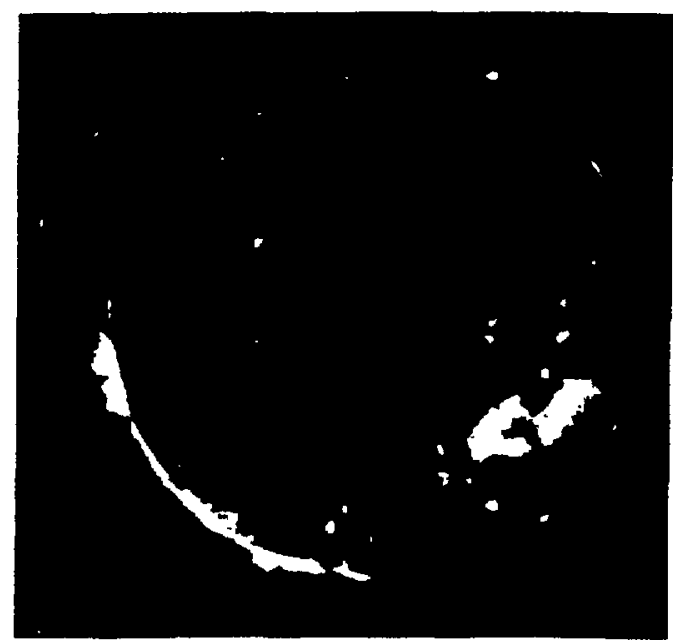

Fig. 4. The solar corona as photographed in the emission of the resonance lines of $F e$ IX at 171 and $\mathrm{Ge} X$ at $174.5 \mathrm{~A}$. The dark bands visible at northern latitudes are due to overlying cool prominences. The lmage corresponds to coronal structures from $0.8 \times 10^{6}$ to $1.4 \times 10^{6} \mathrm{~K}$.

bination as an additional mechanism to reduce the mean lonic charge.

A picture of the Cygnus loop, 6 taken with soft $(8-80$ A) $X$ rays, is shown in Fig. 5. The Cygnus loop is a supernova remant which has expanded to the point where we can look at detalled structure. Another area is revealed in a photograph (Fig. 6) taken with visible light coming from a cooler shell. In the visible, the oxygen rich supernova remnant will look very green in a color photograph because of the large contribution from the 4959 A and $500 \%$ \& 0 III lines. Information of the sort presented in these figures is vital to all, theorles of Stellar evolution.

AC ICPEAC XVI, we shall have two plenary talks on collision physics in relation to astrophysics. At the low energy end, a lecture on "Low Energy Molecular Coliisions with Applications to Interstellar Cloud Problems" by k. Takayanagi, and a lecture covering a recent: very notable higher energy event "Atomic and Molecular Processes in Supernova 1987" by" R. A. HcCray.

\section{Chemically Reactive Collidons}

Chemically reactive collisions, and here I include ion-molecule reactions, also covers a broad range on a logarithmic scale of energies.

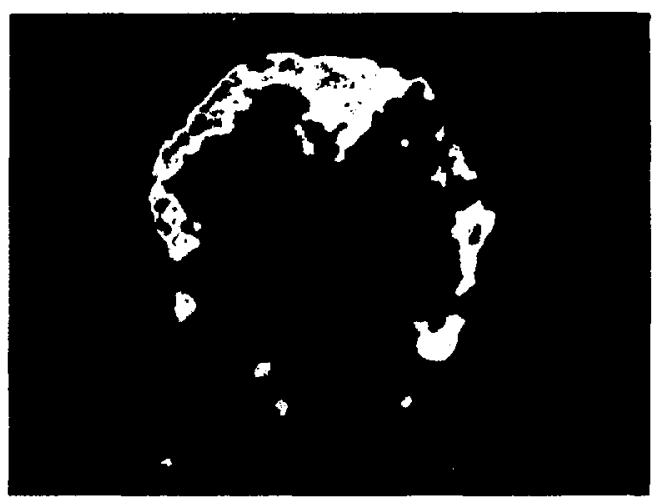

FIG. 5. The Cygnus loop photographed using its emitted soft $X$-ray radiation.

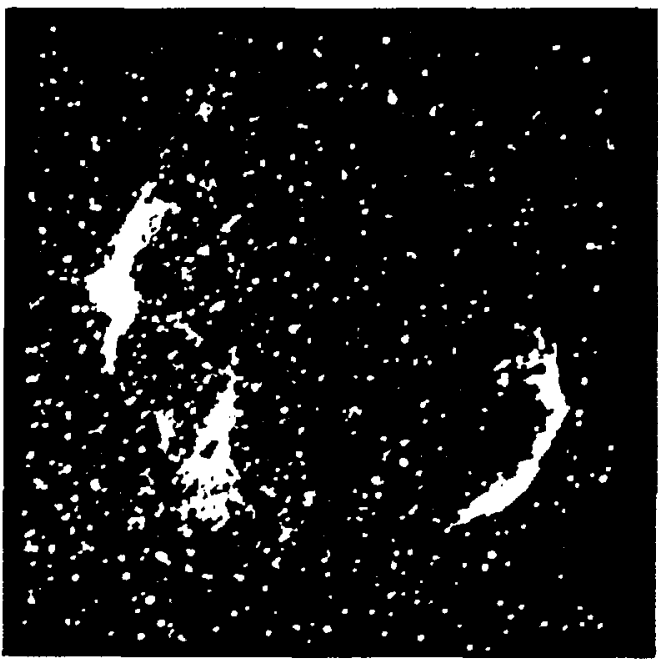

FIG. 6. The Cygnus loop photographed using its emitced green $0^{+} 11 \mathrm{ght}$.

The coldest to my knowledge being the observation of the reactions

$$
\begin{aligned}
\mathrm{He}^{+}+\mathrm{H}_{2} & \rightarrow \mathrm{He}+\mathrm{H}^{+} \mathrm{H}^{+} \\
& \rightarrow \mathrm{He}+\mathrm{H}_{2}^{+}+\mathrm{hv}
\end{aligned}
$$

at $10^{\circ} \mathrm{K}\left(10^{-3} \mathrm{eV}\right)$ in an ton trap by a group at IILA. ${ }^{7}$ it is noteworthy that the reaction of $\mathrm{He}^{+}+\mathrm{H}_{2}$ has been studled at energies up to $i$ MeV, hence 9 orders of magnitude. An entire sympositm on "Colliolong with Cold Particles," chaired by H. Metcalf, will be presented at this conference.

The upper portion of the energy range belongs to hot atom chemistry and ion molecuin reactions. 
The subject of cherical kinetics was liberated from the bulb and the test tube in the rid 1950's with the successful application of molecular beam techniques. 8 Two papers on the subject appeared at ICPEAC II in 1961. One was on "Reactive Scattering of Velocity Selected $K$ Atons" by D. Beck, E. F. Greene, and J. Ross, 9 and the other entitled "Reactive Scattering in Crossed Molecular Beans" by G. K. Kwe1, J. A. Norr1s, J. L. Kinsey, and D. R. Hershbachio discussed the reactions of $\mathrm{CH}_{3} I+K, \mathrm{Rb}$, and $\mathrm{Cs}$. This was, in fact, the first publication from the Hershbach group.

By the mfd-1970's, the scope of the technique was already permitting the investigation of state-to-state chemlcally reactive collisions as illustrated in Fig. 7, which is taken from a 1973 review by Hershbarh. 11 It lllustrates the range of reactions avallable for study at the $t$ ime together with the possible ways of preparing specific reagent states and analysis of product states. The enormous progress that has been made since these early days is exemplified at this conference by the plenary paper of $Y$. T. lee on "Molecular Beain Studies of Cherical Reactions" and the review paper by W. H. Miller on "Recent Developments in the Theory and Application of Quantur, Scattering Theory for Chemical Reactions."

\section{Atmospheric Physics}

Atomic collision processes control the composition of the upper atmosphere and, to a growing extent, also the lower atmosphere. One has to be totally 1lliterate not to be aware of the threats to the lower atmosphere by the massive production of polutants such as $\mathrm{NO}_{x}$ and $\mathrm{O}_{3}$ and threats to the upper atmosphere by the even increasing $\mathrm{CO}_{2}$ concentration with its attendant "Greenhouse Effect" and by the disappearance of the protective ozone layer which is being attacked by free chlorine atoms photolytically produced from chloro-fluoro carbons. The potential ozone disaster was first described by S. Rowland, a hot-atom chenist frow the

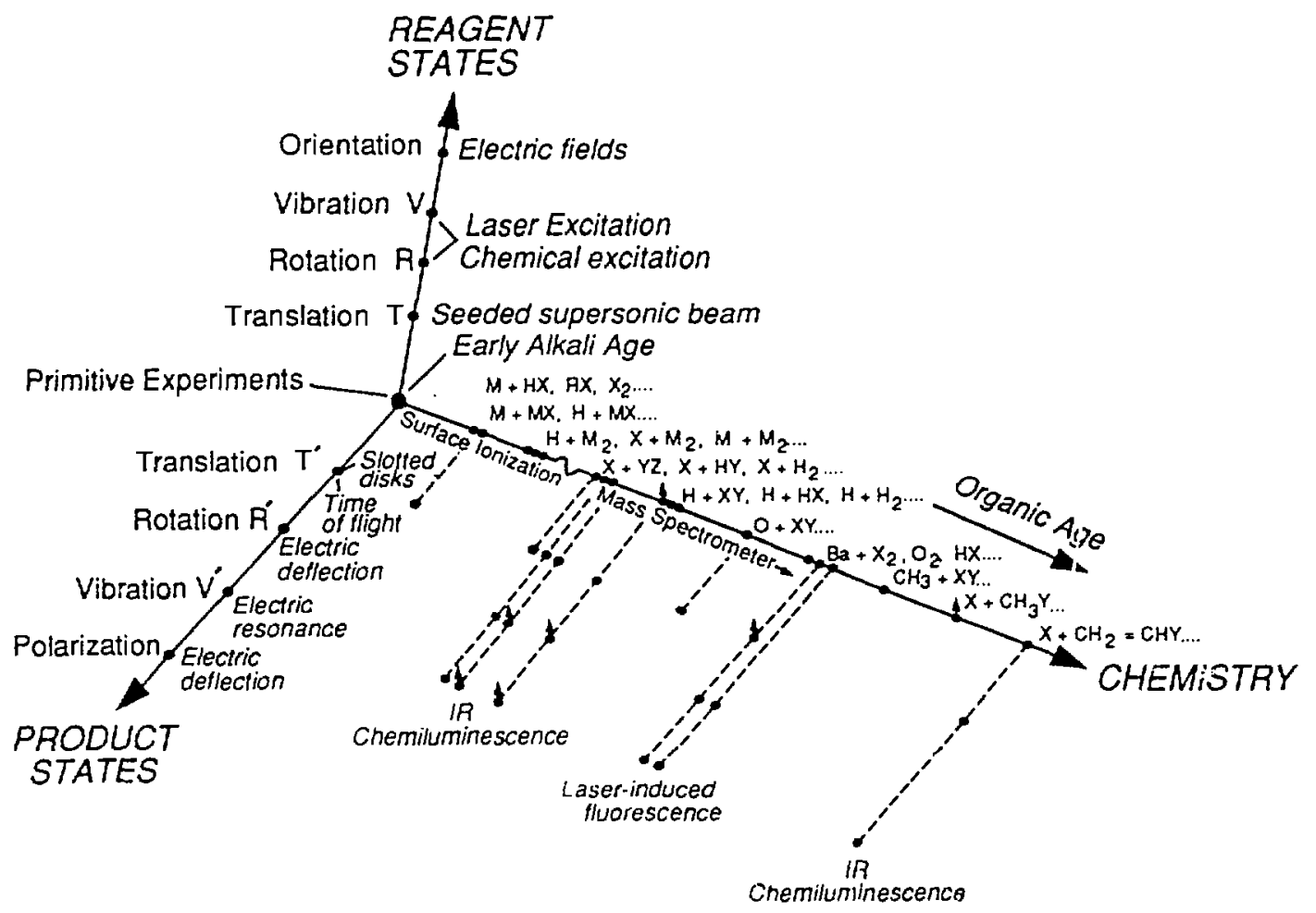

FIG. 7. Danains accesstble to molecular beam chemistry, circa 1973. 
University of California at Irvine almost twenty years ago. The effect is explained by a simple series of binary aron molecule collisions. To determine whether chloro-fluro carbons were cruly a threat or whether competing natural processes would mitigate the effect required careful measurements of a whole serles of reaction cross sections. Needless to say, the impact of these ny threats on the chemical industry, the worlds economy, methods of energy generation, conservation, etc., are potentially enormous and contributions to knowledge by members of our craft may do much to help find solutions to alleviate these problens.

On a more benificent note, we can enjoy the collisional Interactions of electrons exciting th: oxygen red line at $6300 \mathrm{~A}$ as they swirl in the earth's magnetic field 12 and create the red aurora borealis via the $2 \mathrm{p}^{4}-\mathrm{D} \rightarrow 2 \mathrm{p}^{4} \mathrm{p}^{3}$ transition (Fig. 8).

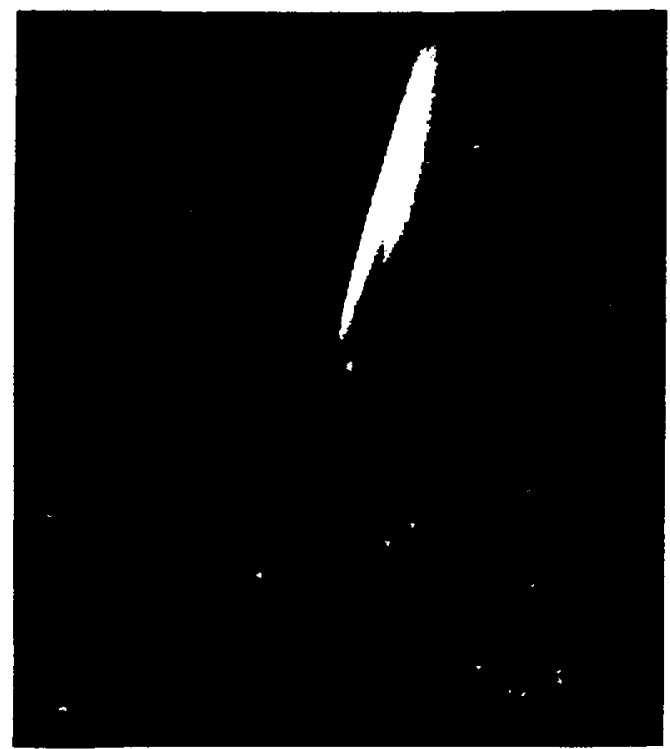

F1g. 8. The red aurora.

\section{E. X-ray Lasers}

As we move up in energy, we enter the region of incompletely tontzed plasmas and hence the area of gaseous electronics. This is an area of enormously broad utility ranging from such mundane things as gas discharge lighting of our streets at the low energy end to the presentiy exotic lasers at the higher end.

As is the case with any normal gas laser, the photons arise from the stimulation of transitions of outer shell electrons with inverted populations.13 Since the energy levels of highly lonized species are further apart, the product photons are in the $\mathrm{X}$-ray region. The population inversion needs pumping, and, because of the short radiative lifetimes involved, this creates special problems. In general, $X$-ray laser schemes utilize atonic collision processes to pump the inversion. The two inversion schenes presently in use are recorabination to inverted states in a cooling plasma and free-electron collisional pumping. In particular, I should like to note that it was necessary to develop a dielectronic recombination theory to explain the kinetics responsible for the strong amplification in the $3 p+3 s \quad(J=2 \rightarrow 1)$ line of neon-like selenium 14 (see below). A review talk on the "Atomic Physics of Soft X-Ray" Lasers" will be presented at this conference by A. V. Hazl.

\section{F. Fission Pragments, Tracks and Stopping Powers}

More complex is the understanding of the interaction of energetic heavy ions, such as fission fragments with the sollds into which they recoil and, in general, the physics of heavy particle tracks. Very recent studies by Schmidt-Böcking and co-workers and by Ron olson on the details of inelasticity and secondary. electron production in such collisions now show great promise to finally understand the phenomena on an atomic basis. 15

\section{G. Fusion}

Even a cursory discussion of the need for atomic collision data in the development of magnetically confined controlled fuston plasmas, as well as inertially confined plasmas, coulu easily take up this entire talk. Numerous reviews and conferences have been devoted to just this subject. Suffice it to say here that any and all information on collistonal processes of electrons, hydrogen atoms, hydrogen molecules, He ions, totally and partially stripped heavy ions in the energy range of a few $\mathrm{eV}^{\mathrm{V}}$ (plasma edge) to tens of $\mathrm{keV}$ (plasma center) are of immediate interest to this extremely inportant technology of the future. Immediately relevant invited papers at this conference include those by A. Bárányt, P. Helplund, R. Schuch, E. Salzborn, and L. Shmaenok.

\section{H. Accelerator Technology}

Particle accelerators are ingtruments with a auch broader range of uses than is generally attributed to them (i.e., riuclear and particle physics). One need only peruse the proceedings of the latest conference or the "Application of Accelerators in Research and Industry"16 vo find in addftion to 52 papers on accelerator 
technology; 95 papers on atomic physics and related phenomena; 20 papers on proton induced $X$-ray analysis (PIXE) and lon microprobes; 36 papers on materials analysis factlities, accelerator mass spectroscopy, Rutherford backscattering and channeling, nuclear reaction analysis, resonant ionization spectroscopy; and 13 papers on radiation therapy, neurosurgery with ion beans (this is only a partial list of papers).

Applications of atomic collision physics to accelerator technology actually begin with ion sources of which many are properly in the domain of gaseous electronics. Negative ion production is Important for tandem Van de Graafs $\{7$ as is multicharged ion production for heavy particle accelerators. In this regard, a number of these wulticharged ion sources, such as Electron Cyclotron Resonance Ion Sources (ECR), Electron Beam Ion Sources (EBIS), and the newly develcped Electron Beam Ion Trap (EBIT) are themselves powerful tools with application to atonic collision physics.

Accelerated ions are often stripped of electrons by collisions in either gaseous or thin solid foils as in tandem electrostatic accelerators for further acceleration at higher charge states, or to achleve lower magnetic rigidity for injection into boosters. A knowledge of the electron capture and loss processes, and equilibrium charge states at the relevant energies is vital for the design paraneters of these systems. 18

Especially in synchrotrons where lons travel enormous pathlengths and espectaliy during the acceleration phase, particles can be lost because of lonlzation or charge capture with background gas. Hence the requisite vacuum conditions and their associated costs are determined from a knowledge of charge-transfer cross sections.

\section{Palr Production and Electron Capture}

As we accelerate to even higher energies, the standard charge-changing cross sections decreas a rapidly, but if we wish to take advantage of the avallable currents, and the kinenatics of storage ring colliders, such as the proposed Relativist1c Heavy Ion Collider (RHIC) at Brookhaven or the hadron Collider at CERN, we encounter some new atomic physics phenomena ramely lepton pair sreation (e.g., electronpositron palrs) and negative lepton capture from the negative continuum.

To quote frow a recent paper by Bottcher and Strayer"19 "One of the mot useful modem probez of hironic matter is the auscciated production and decay of lepton pairs during a collision. In such collisions, lepton-hadron final state interactions are usually small, and hence the leptons carry direct information on the space-time region of creation. Historieally, lepton pair production has been an important tool in collider experiments, in part, because of the special relationship between deep inelastic lepton-hadron scattering and the large mass Dreli-Yan processes provide complementary information on quantum chromodynamics (QCD) in the asymptotic regime. from these experiments have arisen new ideus and phenomena: scaling, chiral and flavor symetry, charm, and a quantitative understanding of a rich meson and baryon spectroscopy."

The problem is that the cross section for lepton creation by non-nuclear electromagnetic (1.e., atomic physics) processes is larger than that for the desired process; hence a detalled knowledge of the cross section for their production, etiergy and angular distributions. The process is siaply described as the formation of a virtual photon which decays by pair production. The venerable and elegant Weizaker-Williams approach to the problem is both approximate and perturbative and does not directly yleld angular and energy distributions. More exact perturbative calculations have recently been carrled out 19 and they, of course, differ somewhat from the Weizaker-Williams result. More disturbing, however, are the results of a non-perturbative calculation, 20 (see Fig. 9) which predicts differences of as much as factors of, e.g., 100 at RHIC energies (100 GeV/nucleon).

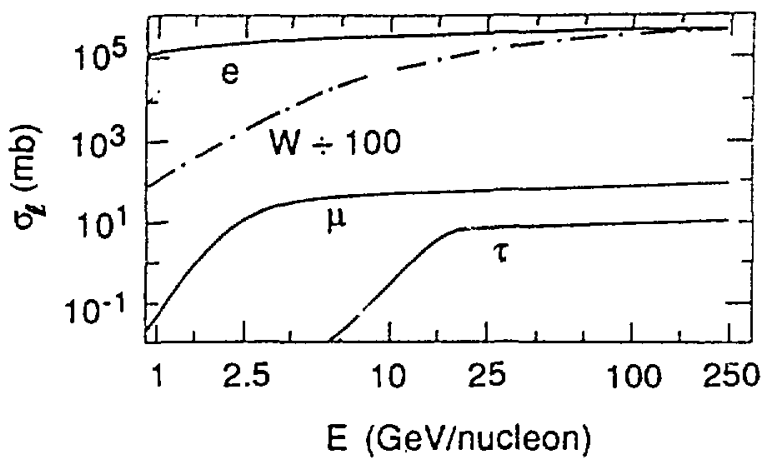

FIG. 9. Predicted croes sectiong (millibarns) for $e^{ \pm}, \mu^{ \pm}$, and $\tau^{ \pm}$pelr production for $\mathrm{U}^{92+}+\mathrm{U}^{92+}$ collisions $V$ s $\gamma$. For RHIC, $\gamma$ lut for CERN SPS (200 GeV/c/amu) on a fixed carget. $\gamma=10$. The solld ine for te from nonpertur. bative calculation is sompared with the Welzaker-Williams calculacion $(W+100)$. 
Since the pair is created in the immediate vicinity of the projectile nucleus, there is a strong posibility that the electron (negative lepton) may be captured. Hence the tonic charge changes and, if this occurs in a storage ring. the particle is lost. In fact, this process may be the limiting factor for containment times in such devices. Some detailed, but perturbative calculations have recently been carrlea out ( $F 1 \mathrm{~g} .10), 21$ but thus $\mathrm{f}$ ar no experiments have been performed. Clearly, this possible polson for particle physics poses a very interesting problem in atomic physics and proposals have been wounted for experiments on $e^{ \pm}$pair production and electron capture at SPS (200 GeV/amu CERN), the AGS (20 GeV/amu - Brookhaven), and Bevelac (1 GeV/amu - Berkeley).

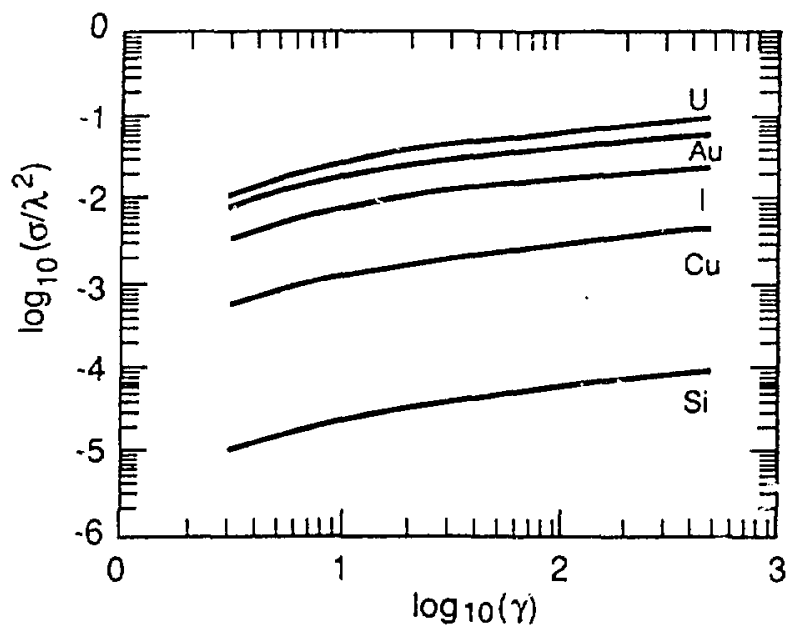

FIG. 10. Capture cross sections for symmetric $A_{2}+A_{z}$ collisions, scaled with respect to $\lambda^{2}=1.49 \mathrm{~Kb}$. Curves correspond as labeled, to the lons $A(z)=S 1(14), C u(29), I(53)$, $\mathrm{Au}(39)$, and $U(92)$, e.g., for $\mathrm{U}^{92+}$ at RHIC energies $\sigma_{\mathrm{c}} \sim 100 \mathrm{~b}$.

\section{J. Reverse KInematics}

The remaining bar on Fig. 3 "reverse kinematics" is not a science or a technology, but a name applied by nuclear physiclsts to a technique in which one uses high laboratory energy techniques to study collisions at lower center-of-mass energies; for example, an accelerated arbon ion 19 shot at a hydrogen target rather then a proton at a carbon target. Such kinematic trlcks are in extensive use by atomic. collision physicists as we shall gee below.

\section{DIELECTRONIC PROCESSES IN ELECTRON-ION, ION-ATOM, AND ION-SOLID COLLISIONS}

Up to this point, we have been discussing the general utility of atomle collision physics in other flelds of endeavor. At this point, I would like to become nore specific and investigate the interaction between varlous and seemingly diverse branches of atomic collision physics in investigating a single problen, i.e., dielectronic excitation.

First, we will consider dielectronic reconbination which occurs in collisions of lons with free electrons. This process is the dominant recombination mechanism in hot heavy-ion plasmas and, as we have indicated above fs important to understand for such diverse applications as $X$-ray lasers and coronal temperatures. Second, we will examine resonant electron transfer and excitation (RTE) which occurs when an ion col11 des with an almost free electron weakly bound to an atom. Finally, we will take a look at recent studies of dielectronic excitation in crystal channels whlch have been shown to behave as dense Fermi electron targets.

Dielectronic recombination (DR) is initiated when a continuum electron excices a previously bound electron and in so dolng loses just enough energy to be captured itself into a bound state $(n l)$. The latter process results in a doubly excited ion (dlelectronic excitation) in the next lower charge state which may either auto-ionize or emit a photon resulting in a stablized recomblnation (F1g. 11). Thus, for an ion $A$ of charge state $q$ in initial state $\alpha$, the $D R$ process may be written:

$$
\begin{aligned}
A^{q+}(\alpha)+e^{-}\left(k, \ell^{\prime}\right) \neq & {\left[A^{(q-1)+*}(\beta, n \ell)\right] \star \star * } \\
& {\left[A^{(q-1)+}(\alpha, n \ell)\right]^{*}+h v . }
\end{aligned}
$$

It should be noted here that the first step, 1.e., the Eormation of the dielectronically excited state is the reverse of the Auger process. Experimental results in DR were first reported In 1983 at the Berlin ICPEAC in a "hot topic" symposium. Work has proceeded apace since then but two techniques which are giving qualitative improvements in the data have just come into frultion; these will be reforted in invited talks by $P$. Huelplund, R. Schuch, and A, Wolf, Both techniques utilize a merged electron-ion beam rechnique, 22 one in a single pass experiment ${ }^{23}$ and the second as part of a cooled heavyfon storage ring. 24

The Aarhus experimental getup is described in a paper by 9 . Huelplund in this volume. In the example we discuss here, ${ }^{23}$ a beas of $20-\mathrm{MeV} \mathrm{O}^{6+}$ lons 1 merged with an electron beam 


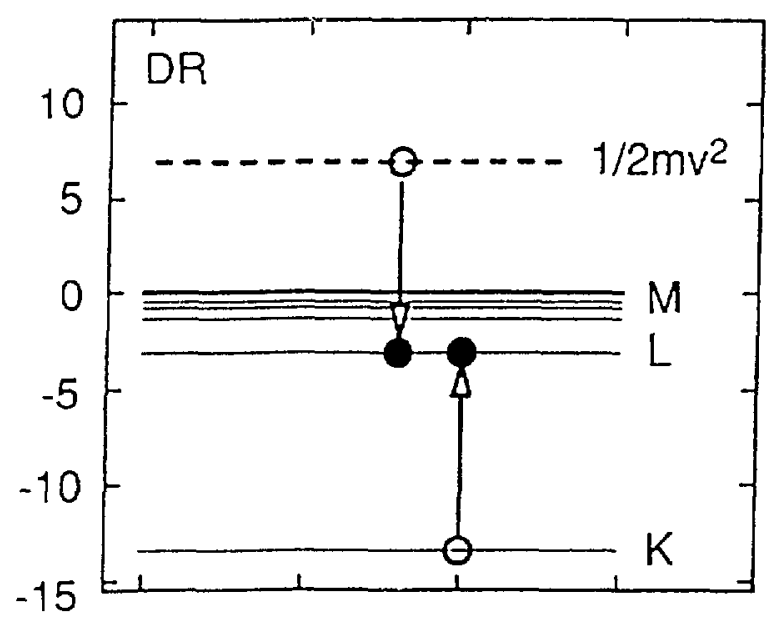

FIG. 11. Dielectronic excitation of a hydrogenic ion to a KlL state. Radiative relaxation leads to dielectronic recombination.

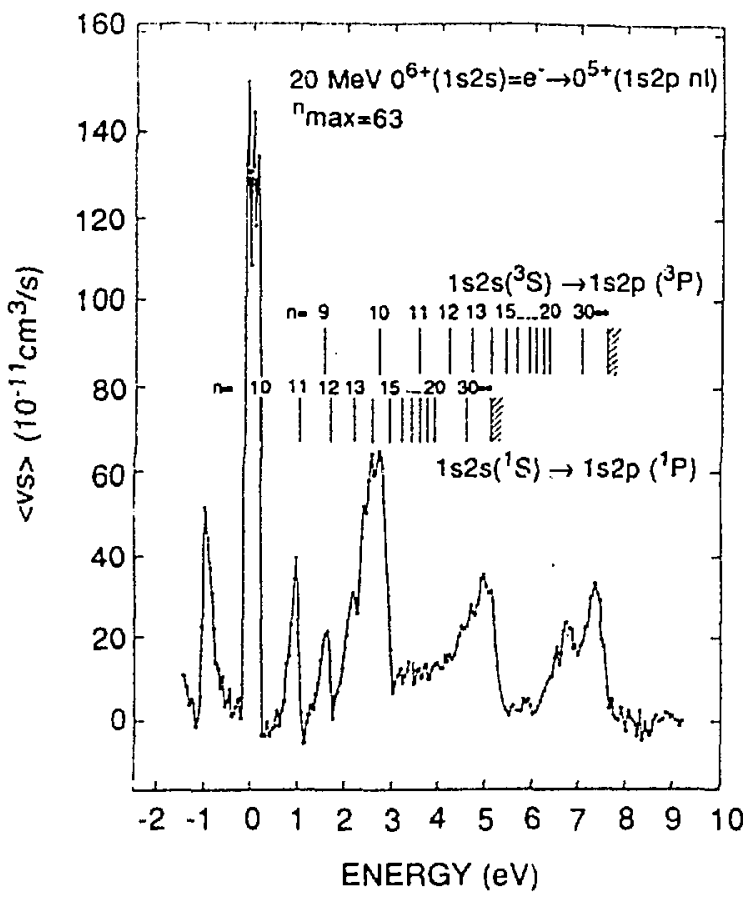

Fig. 12. Dielectronic recombination spectrum of $0^{57}$ ( $1 \mathrm{~s} 2 \mathrm{~s}$ ) obtained in a single pass merged electron-ion beam experiment (see Ref. 23).

whose velocity is varied to give relative velocities 1.n the $0-20 \mathrm{eV}$ range. The resultant recombination (to form $0^{5+}$ ) spectrum shown in Fig. 12 arises from the $1 \mathrm{~s} 2 \mathrm{~s}$ metastable component of the beam via the transitions $1 \mathrm{~s} 2 \mathrm{~s}\left({ }^{1} \mathrm{~s}\right) \rightarrow 1 \mathrm{~s} 2 \mathrm{p}\left({ }^{1} \mathrm{p}\right)$ and the $1 \mathrm{~s} 2 \mathrm{~s}\left({ }^{3} \mathrm{~s}\right) \rightarrow 1 \mathrm{~s} 2 \mathrm{p}\left({ }^{3} \mathrm{p}\right)$ and ( ${ }^{1} P$ ) states. Note that although the fon beam energy in the laboratory is $20 \mathrm{NeV}$, the center-of-mass energy ranges from $0-10 \mathrm{eV}$ with a resolution of $\sim 0.15 \mathrm{eV}$.

The completion of heavy ion storage ring profects will lead to many new and dramatic advances in heavy 1on atomic physics. The merged electron beam exists as an integral part of the ring whers it is used for "cooling" the stored beam. ${ }^{24}$ One advantage of the storage ring is the enormous increase in effective current and attendant luminosity obtained by circulating the same particles through the thin target at frequencies of up to a megahertz. The first results on DR obtained from the Heidelberg ring $^{23}$ (the first of the completed profects) will be discussed in invited papers by $R$. Schuch and by $A$. Wolf.

Another experiment which gives related results involves electron transfer plus excitation ( $T E$ ) in ion-ator collisions. In this case, Instead of a truly free electron, we use an atomic target which has weakly bound (almost free) electrons with orbital velocities $v_{e}<<$ $v_{1}$, where $v_{i}$ is the ion velocity. This process has been dubbed "Resonant Transfer and Excitation" (RTE). 25 Here, e.g.,

$$
A^{q+}(\alpha)+\mathrm{He} \rightarrow\left[A^{(q-1)+}(\beta, n \ell)\right]^{\star \star}+\mathrm{He}^{+}
$$

the doubly excited state can then relax via Auger or radiative decay

$$
\begin{aligned}
{\left[A^{(q-1)+}(\beta, n l)\right]^{* *} } & \rightarrow A^{q+}(\alpha)+e_{A} \\
& \rightarrow\left[A^{(q-1)+}(\alpha, n l)\right]^{*}+h v_{1}
\end{aligned}
$$

Further, che singly exclted state can relax with the emission of a second $x$ ray

$$
\left[A^{(q-1)+}(\alpha, n \ell)\right]^{*}+A^{(q-1)+}\left(\alpha, \alpha^{\prime}\right)+h \nu_{2} .
$$

The end results are either an Auger electron which contains information on the ne state of the originating doubly excited state, or an ion of decreased charge and two photons. Experiments have been carrled out by measuring the energy dependence of the Auger electron spectra (RTEA); charge capture in coincidence with $X$ raye ( $R T E X)$; or two $X$ rays in coinctdence (RTEXX). In all cases, the relative coiliston energy is scanned by varying the energy of the 1on beam. RTE will be discusaed in papers by Graham, Hahn, and Schuch in a symposium 
chaired by J. Tanis, who ploneered this technique.

As an example, consider the results of one experiment carried out at the Berkeley Super HILAC by Graham et al. 26 shown in Fig. 13. The Ion used was $\mathrm{He}-11 \mathrm{ke} \mathrm{Cal8+}$ and the target was $\mathrm{H}_{2}$. Here we can see a signiflcant effect of RTE on the cotal electron capture cross section $\left(\sigma_{q}, q-1\right)$. The effect is much enhanced in the measurement of a $K-X$ ray in colncldence with the formation of $\mathrm{Ca}^{17+}$. The lower energy bump, in this case, corresponds to a KLL excitation. The width of the peak is the result of a fold of the momentua distribution (Compton profile) of the bound electrons in the $\mathrm{H}_{2}$ molecular target with the $\mathrm{KLL}$ resonance lines.

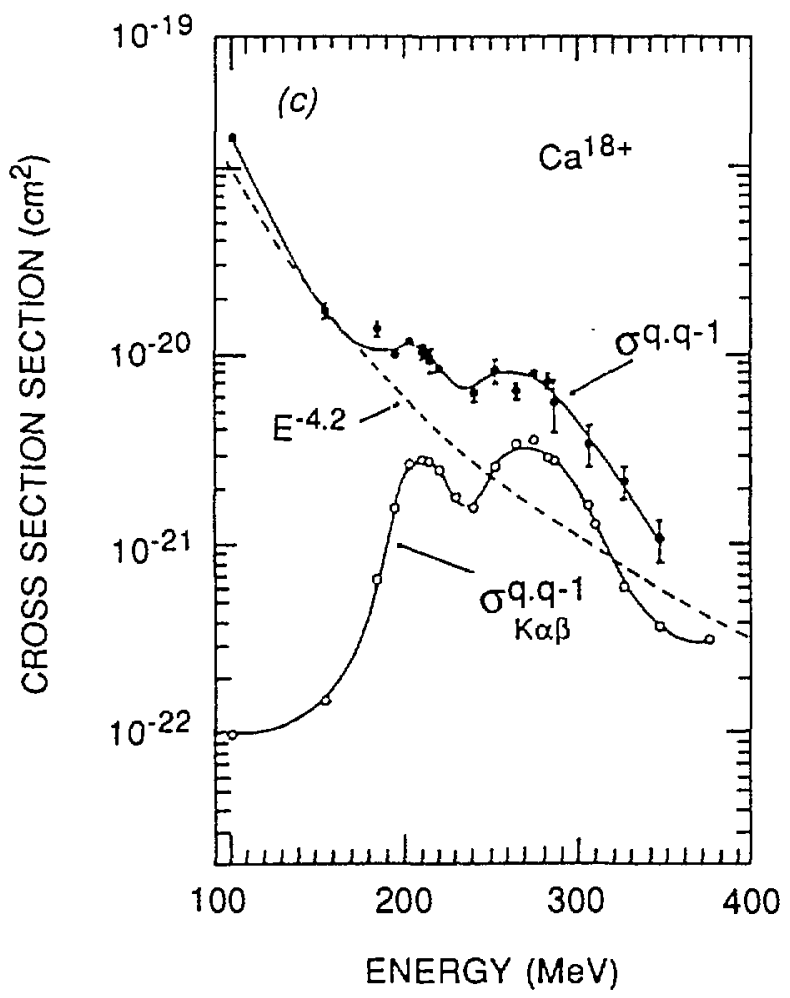

EIG. 13. Upper curve, measured caprure cross section for $\mathrm{Ca}^{2}+$ on $\mathrm{H}_{2}$ showing contributions from RTE. Lower curve, capture measured in colncidence with calcium KX ray (see Ref. 26).

Dielectronic excitation processes have now aiso been studied in crystal channels. Energetic ions traveling through crystals at small angles to low index directions may be steered to avold small Impact parameter collistons with the atomic cores of the lattice atoms ("channeling) and interact directly only with loosely bound electrons. 27 For lon velocicles $v_{1} \gg v_{f}$, where $v_{f}$ is the velocity of the target Fermi electrons, the penetrating ion may be viewed as being bombarded by a flux of electrons moving at velocity $v_{1}$.

Two invited papers at the present conference deal with consequences of this effect: one by J. C. Polzat on "Energy Loss and Charge Exchange Processes of High Energy Heavy Ions Channeled in Crystals" and the second by $\mathrm{N}$. Claytor on the "Measurement of Electron Impact Ionization of $\mathrm{U}^{88+}-\mathrm{u}^{91+\prime}$ in which $\sim 500 \mathrm{MeV} / \mathrm{amu}$ ions are made to collide with the electrons in a crystal channel ("reverse kinematics"). If this assumption is quantitatively correct, lons traveling through this medium at velocities equivalent to the electron velocities required for sharply varying piocesses, such as dielectronic or direct excitation of an electron bound to the ion, should experience events similar to those in a hot dense $\left(>_{10}^{23}\right.$ electrons $\left./ \mathrm{cm}^{3}\right)$ plasma, but with a relatively narrow electron energy distribution, 1.e., a Fermi distribution as against a Maxwel1-Bolzman distribution at the temperature necessary to carry out these excltation processes.

In vacuum under single collision conditions, the doubly excited state formed by dielectronic excitation would decay, as above, either by an Auger process or by radiative stabilization via two photons. The final result being the production of an lon of reduced charge state and two photons. However, if the state is created in a dense electron medium (i.e., a dense plasma or a crystal channel), secondary collisional processes leading to further excitation and lonization can come into play and may everi dominate.

Take, as an example, the dielectronic excitation of a hydrogenic ion to a $\left[2 \mathrm{p}^{2}\right]^{\star \star} \mathrm{KLL}$ resonance (see e.g., Fig. 11). This state could be collisionally ionized to $[2 \mathrm{p}]^{\star}$ or $1 \mathrm{t}$ could be excited to a $[2 \mathrm{p} 3 l]^{\star \star}$ state. The excitation cross section from a given $n$ state to an $(n+1)$ state is larger than that for direct ionization, but the fonization cross sections for high $n$ states arn so large that for $n>3$ in the cases under consideration, ${ }^{28}$ the electron can be considered as removed from the ion by either excitation or lonization. The remaining $2 \mathrm{~F}$ electron can then elther radiate to ls or undergo a sinilar collisional excitacion or lonization. Thus, one possible path leads the lonization to form a bare nurleus at single collision energies below the first excltation potential. As an exanple, the resulta for $\mathrm{Ca}^{10+}$ lons $(150-330 \mathrm{MeV})$ are shown in Fig. 14. The Calot ion beam passed through the $\langle 110\rangle$ axis of a $1.2 \mathrm{\mu m}$ thick $\$ 121 \mathrm{con}$ 

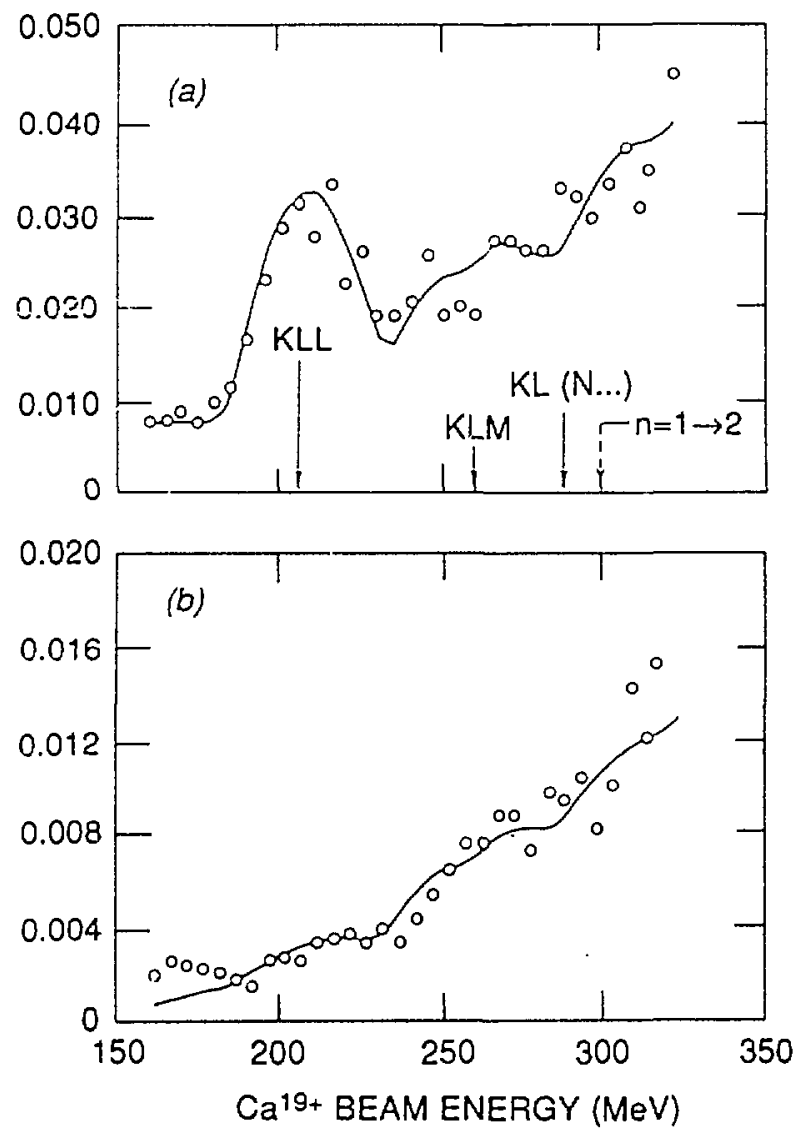

FIG. 14. (a) Yield of H-like $K_{a g}$ calcium $X$ zays and (b) charge fraction of $\mathrm{Ca}^{20+}$ ions as a function of $\mathrm{Ca}^{29+}$ ion energy incldent on a $\langle 100\rangle$ channel in $\mathrm{Si}(1.2 \mu \mathrm{m}$ thick) the smooth curves are calculated from the appropriate rates.

crystal. Measurements were made of (a) the emerging charge-state distributions using electrostatic deflection and a solid-state position-sensitive detector, and (b) the X-ray spectra using a Si(L1) detector which can resolve $H-l i k e \mathrm{~K}_{\alpha}$ from the He-like $\mathrm{K}_{\alpha}$ lines.

In Fig. 14a, the yield of H-like $k_{\alpha} x$ rays is shown as a function of ion energy. The features correspond to dielectronic excitation to KLL, KLM, KLN, etc., and at $300 \mathrm{MeV}$ to direct is $\rightarrow 2$ excltation. The calculated absolute magnitudes of these contributions are obtalned frow the appropriate rate equations contalning radiat ive and Auger rates and collision cross sections. They are then folded with the appropriate Fermi distribution. Using the same rate coefficient as those used for the $x$-ray ylelds shown in Fig. 14a, the yield of baris $\mathrm{Ca}^{20+}$ ton is calculated and compared with the data in Fig. 14b. (Dielectronic excitation in channels is discussed in contributed papers 137 and 138 ).

\section{CODA}

In this paper, I have tried to point out not only some features of the utility and ubiquity of atomic collision physics, but also the response of the ICPEAC community in meeting the challenges created by the needs of the general sclentiflc and technological community. The great utility or atomic collision physics is, however, both a strength and a weakness; the weakness being the perception of our fleld as an adjunct of other disciplines toward which its results are applicable rather than as a separate discipline in and of itself.

The object of the latter part of the talk was to point out the close inter-relationship between seemingly disparate branches of our science, 1.e., 1on-atom, electron-ion, and ionsolid collisions. All of these are presently represented at ICPEAC. This demonstration was, in part, intended as a response to those who believe that ICPEAC is now too large and ought to be broken up into separate subdiscipline conferences .... When I was a lad growing up In New York, the New York Yankees' baseball ream was by far the best in the world habitually winning the "Aqerican League Pennant" and the "World Series." Fans who favored other teams were heard to shout "Break up the Yankees!" Break up the Yankees? .... Never!

This research was sponsored by the U.S. Department of Energy, Office of Basic Energy Sclences, Division of Chemical Sciences under Contract No. DE-ACO5-840R21400 with Martin Marietta Energy Systens, Inc.

\section{REFERENCES}

1. See e.g., Atomic Physics in Nuclear Experimentation in Annals of the Israel Physical

Soclety 1, B. Rosner and R. Kalish, eds., 1977.

2. Applied Atomlc Collision Physics, H. S. W. Massey, E. W. McDantel, and B. Bederson, eds., Vol. 4 Condensed Matter, S. Datz, ed., Acaderic Press, New York, 1983.

3. A. Dalgarno, "Applications of Atomic Collision Physies to Astrophysics" in Physics of Electronic and Atomic Collistons, S. Date, ed., North-Holland Press (1981), pp.

4. A. B. Walker, T. W. Barbee, R. B. hoover, and J. K. Lindblon, Sclence 241, 1781 (1988). 


\section{REFERENCES (contd)}

5. A. Burgess, Astrophys. J. 139, 776 (1964).

6. W. P. Blalr, J. C. Raywond, J. Danziger, and F. Matceucci, Astrophys. Journal 338, 812 (1989).

7. M. Schaer, S. Jefferts, S. Barlow, and 6. Dunn, J. Chem. Phys. (in press).

8. E. H. Taylor and S. Datz, J. Chem. Phys. 23, 1711 (1955).

9. D. Beck, E. F. Greene, and J. Ross, Proceedings, Second Int'l Conf, on the Physics of Electronic and ALomic Collisions, Books of Abstracts, W. A. Benjamin and Company (1961), p. 94.

10. G. H. Kwei, T. A. Norris, I. L. Kinsey, and D. R. Herschbach, Proceedings, Second Int'l Conf. on the Physics of Electronic and Atonic Collisions, Book of Abstracts, W. A. Benjamin and Company (1961), p. 98.

11. D. R. Herschbach, Farad. Dis. Chem. Soc. 55,233 (1973).

12. See e.g., D. R. Bates, "Alrglow and Auroras" in Applied Atomic Collision Physics, H.S.W. Massey, E. W. McDaniel, and B. Bederson, eds., Academic Press 1, 152-200 (1982). [Photo by Al McNeal, University of Alaska]

13. See e.g., P. Jaeglé, J. Physique 48, C9 (1987).

14. B. L. Whitten, A. U. HazI, M. H. Chen, and P. L. Hagelstein, Phys. Rev. A 33, 2171 (1986).

15. See e.g., U. Ramm, S. Schmidt, H. Schwidt-Böcking, G. Kraft, and R. E. Olson, GSI Scientific Report No. ISSN 0174-0184, p. 205 (1988).

16. Nucl. Instru, Meth. Phys. Res. B 40/41, (1989).

17. G. D. Alton, c.f. Ref. 2, pp. 44-171.

18. H. -D. Betz, c.f. Ref. 2, Pp. 2-43.

19. C. Bottcher and M. Strayer, Phys. Rev* D 39, 1330 (1989).

20. C. Bottcher and M. R. Strayer, Nucl. Instrum. Meth. Phys. Res. B 31, 122 (1988).

2i. M. Rhoades-Brown, C. Bottcher, and $M$. R. Strayer, Phys, Rev. A (in press).

22. P. F. Dittner, Physica Scripta T22, 65 (1988).
23. L. H. Andersen, H. Knudsen, P. Huelplund, and P. Kvistgaatd, Phys. Rev. Lett. 62, 2656 (1989).

24. R. Schuch, Nucl. Instrum. Meth. Phys. Res. B $24 / 25,11(1987)$.

25. J. A. Tanis, E. M. Bernstein, w. G. Grahan, M. Clark, S. M. Shafroth, B. M. Johnson, K, K. Iones, and $M$. Meron, Phys. Rev. Lett. 47, 1325 (1982).

26. W. G. 'iraham, E. M. Bernstein, M. W. Clark, J. A. Tanis, K. H. Berkner, P. Gohil, R. J. McDonald, A. S. Schlachter, J. W. Stearns, R. H. McFarland, T. J. Morgan, and A. Müller, Phys. Rev. A 33, 3591 (1986).

27. "Heavy Ion Channeling," S. Datz and C. D. Moak, in Heavy Ion Science, Vol. 6, D. A. Bromley, ed., Plenum Press, 1985, pp. 169-240.

28. S. Datz, C. R. Vane, P. F. Dittner, J. P. Giese, J. Gonez del Campo, N. L. Jones, H. F. Krause, P. D. Miller, M. Schulz, H. Schöne, and T. M. Rosseel, Phys. Rev. Lett. (in press). 